\title{
Load-Measuring Pot Bearing with Built-In Load Cell -Part II: Fatigue Performance and Experimental Temperature Correction
}

\author{
Jeong-Rae Cho, Young Jin Kim*, Jong-Won Kwark, Sung Yong Park, \\ Won Jong Chin, Byung-Suk Kim \\ Structural Engineering Research Division, Korea Institute of Construction Technology, Goyang-Si, Korea \\ Email: chojr@kict.re.kr, *yjkim@kict.re.kr, origilon@kict.re.kr, sypark@kict.re.kr, wjchin@kict.re.kr, bskim@kict.re.kr
}

Received September 4, 2013; revised October 4, 2013; accepted October 11, 2013

Copyright (c) 2013 Jeong-Rae Cho et al. This is an open access article distributed under the Creative Commons Attribution License, which permits unrestricted use, distribution, and reproduction in any medium, provided the original work is properly cited.

\begin{abstract}
This paper presents the results of fatigue performance tests performed up to 10 million cycles on a load-measuring pot bearing with built-in load cell to verify its field applicability and proposes an empirical temperature correction formula. In Part I of this work, various measurement performances of the load-measuring pot bearing were evaluated through static and dynamic loading tests. Bridge bearings are subjected to the effect of fatigue caused by the repeated application of moving loads and exposed to harsh site conditions including cold and hot weathers differently to laboratory conditions. Accordingly, the durability of the load-measuring pot bearing with built-in load cell shall be secured and the environmental effects like temperature shall be minimized for its application on field. This study conducted fatigue tests up to 10 million cycles on a load-measuring pot bearing with the capacity of $1000 \mathrm{kN}$ to examine eventual degradation of the measurement accuracy with respect to the number of fatigue loading cycles. In addition, the experimental temperature correction procedure is proposed to obtain the temperature correction formula enabling to correct the effect of temperature on the load measurement.
\end{abstract}

Keywords: Pot Bearing; Load-Measuring; Load Cell; Fatigue; Temperature Correction

\section{Introduction}

Cho et al. [1] (referred simply as Part I hereafter) proposed the design concept of a load-measuring pot bearing with built-in small size button-type load cell inserted in the base plate of the bearing and examined experimentally its measurement accuracy and the possibility to achieve dynamic measurement. This study evaluates the durability of the developed bearing for its application in real bridge sites and suggests an empirical temperature correction formula.

Since the load cell presents a load supporting structure in which the strain is converted into an electric signal by a strain gauge attached to the structure, the load cell secures outstanding durability when fabricated as a structure enabling sufficient protection. Bridge bearings shall in general secure durable performance for more than 20 years and are subjected to the repeated application of traffic loads. This means that the load cell inside the

\footnotetext{
*Corresponding author.
}

load-measuring pot bearing presented in Part I is exposed to conditions significantly harsher than those encountered by ordinary measurement devices. The Highway Bridge Design [2] requires the bearings to secure fatigue durability by performing fatigue performance tests up to 2 million cycles. This study conducted fatigue tests up to 10 million cycles to evaluate the stability of the bearing itself and its accuracy as load-measuring device with respect to the number of fatigue cycles accounting for the more severe conditions.

Besides, the load cell itself is fabricated with a built-in temperature correction circuit enabling it to measure the loads regardless of the changes in the ambient temperature. However, the load-measuring pot bearing developed in Part I includes an elastomer disk that is extremely temperature-dependent as central medium transferring the load to the built-in load cell. This situation requires to evaluate the measurement accuracy with respect to the temperature and to apply a temperature correction formula if necessary. This study suggests an empirical tem- 
perature correction procedure to obtain the temperature correction formula adapted to the load-measuring pot bearing with built-in load cell.

\section{Fatigue Test}

Fatigue test performed on bridge structural elements like the bearing shall be executed by applying a fluctuating loading up to the number of cycles specified in the design code considering the vehicle loads with reference to the permanent loads of the bridge. The Highway Bridge Design Code specifies the fatigue durability with reference to 2 million cycles [2]. However, this study conducted fatigue tests up to 10 million cycles accounting for the more severe conditions [3,4].

Bridge bearings supporting the superstructure (permanent loading state) are basically loaded up to $40 \%-60 \%$ of their capacity and experience additional varying loads due to traffic. Here, the load level induced by the permanent loads is set to $60 \%$ and the fluctuation of the load due to the vehicle loads is set to $15 \%$ to conduct the fatigue test on the $1 \mathrm{MN}-\mathrm{L} 1$ specimen with capacity of $1000 \mathrm{kN}$ of Part I. Concretely, the loading history of the planned fatigue test has $P_{\min }=500 \mathrm{kN}$ and $P_{\text {max }}=750 \mathrm{kN}$ as shown in Figure 1. The actual test proceeded by disposing a load cell for the measurement of the load applied on the bearing and the load-measuring bearing between two steel blocks as shown in Figure 2, and by applying the additional dynamic fatigue load by means of an actuator after having applied a constant load through steel bars loaded by the hydraulic jack of which pressure has been maintained by means of the hydraulic nut. The load applied by the hydraulic jack is $500 \mathrm{kN}$, and the additional load of about $200 \mathrm{kN}$ is applied by a dynamic actuator with capacity of $250 \mathrm{kN}$ so as to achieve the intended load level of $500 \mathrm{kN}$ to $750 \mathrm{kN}$ to be experienced by the bearing. Figure 3 plots the fatigue load actually applied on the load-measuring bearing and measured by the load cell disposed above the bearing. The reason for the application of a pre-load using the steel bars and the hydraulic jack instead of applying the load

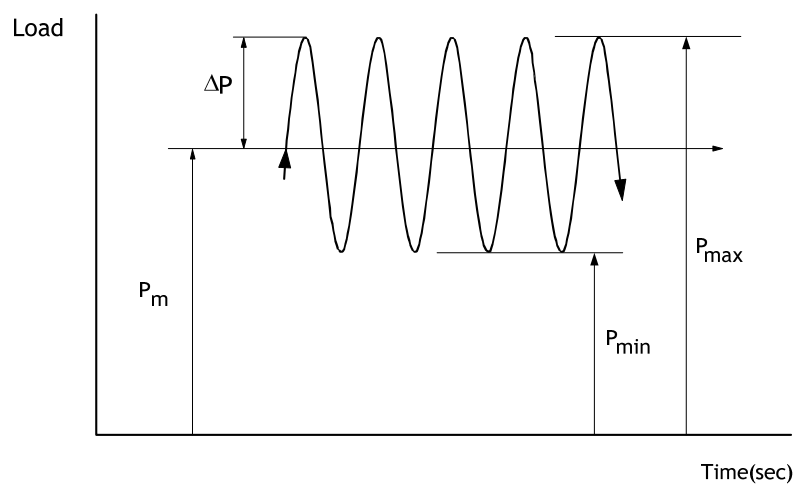

Figure 1. Loading history of fatigue test.
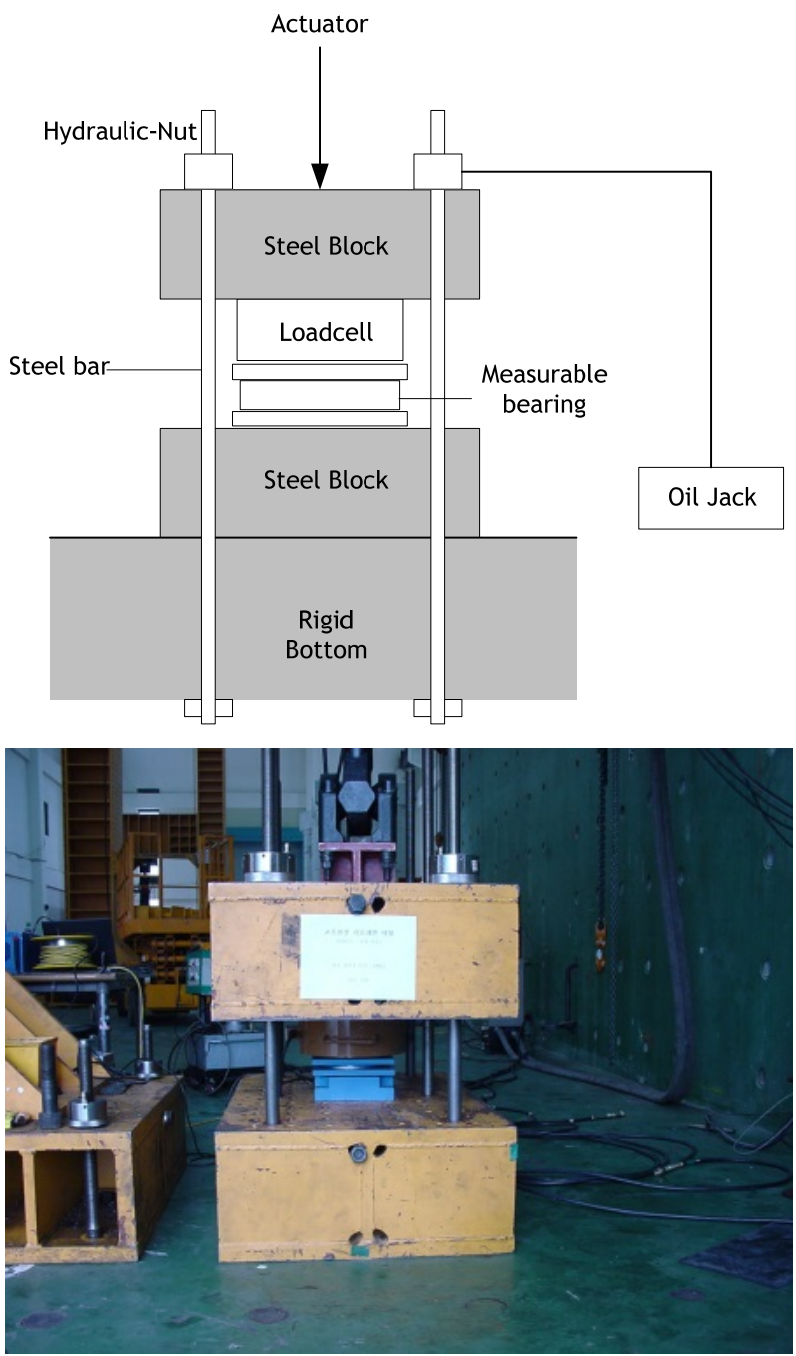

Figure 2. Conceptual scheme of the load-measuring pot bearing with built-in load cell.

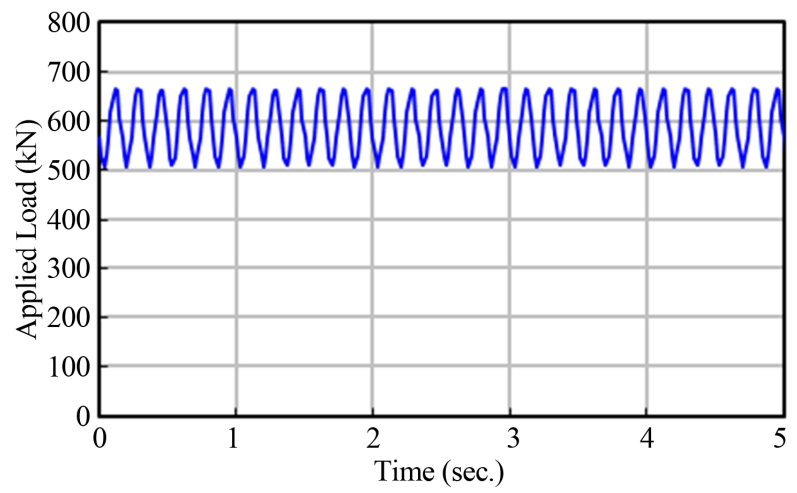

Figure 3. Load applied directly on the specimen during the fatigue test.

directly using a dynamic actuator with capacity of 1000 $\mathrm{kN}$ is due to the durability of the dynamic actuator that has to withstand 10 million cycles of fatigue loading at a high level of 500 to $750 \mathrm{kN}$. 
The stability of the bearing itself and its accuracy as measuring device shall be evaluated according to the number of fatigue loading cycles through fatigue test. To that goal, static loading test was performed by applying the load using the hydraulic jack illustrated in Figure 2 by steps of 500,000 cycles or 1 million cycles after the application of 2 million fatigue loading cycles. Since this test uses a hydraulic jack, loading cannot be applied up to the maximum load at constant speed as done in the static loading test of Part I. Figure 4 plots the test results with respect to the major fatigue cycles. The solid lines represent the values measured by the external load cell in Figure 2 and correspond to the load applied on the loadmeasuring bearing. The dashed lines represent the load obtained by converting the signals measured by the small size built-in load cell inserted in the bearing using the conversion formula for specimen 1MN-L1 derived in Part I and expressed here in Equation (1).

$$
F=456.2208 f-66.4447,
$$

where $f$ is the load $(\mathrm{kN})$ measured by the $5000 \mathrm{kN}$-load cell inserted in the bearing; and, $F$ is the vertical load $(\mathrm{kN})$ applied on the bearing that is the load converted from the measured load.

Figure 5 plots the error rate obtained by dividing the error defined as the difference between the actually applied load and the converted load by the capacity of the bearing according to the number of fatigue cycles. The error rate of specimen $1 \mathrm{MN}-\mathrm{L} 1$ that reached $0.39 \%$ in the static loading test in Part I is seen to increase with larger number of cycles. The error rate remains below $1 \%$ until 4 million cycles and exceeds $1 \%$ after 5 million cycles to reach $2.77 \%$ at 8 million cycles but stands within $2 \%$ at
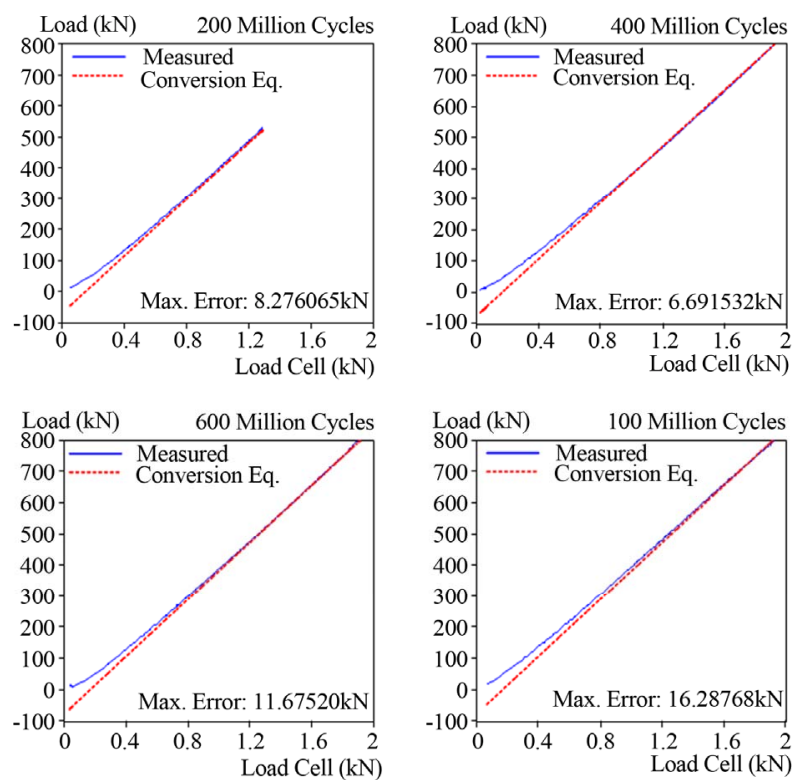

Figure 4. Fatigue test results.

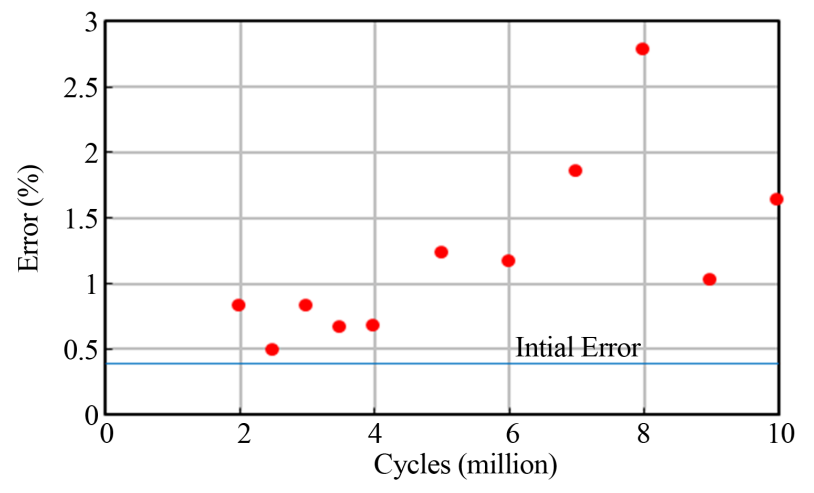

Figure 5. Variation of the error according to the number of fatigue cycles.

the whole between 5 million to 10 million cycles. Considering that the low accuracy load cell secures an accuracy of $1 \%$, these values verify that the error has a level enabling the application of the load-measuring pot bearing for real measurement.

\section{Experimental Temperature Correction}

The load cell itself is fabricated with a built-in temperature correction circuit enabling it to measure the loads regardless of the changes in the ambient temperature. However, the load-measuring pot bearing developed in Part I include an elastomer disk that is extremely temperature-dependent as central medium transferring the load to the built-in load cell. Following, need is to evaluate the effect of the temperature.

The characteristics of the variation of temperature records can be overcome by suggesting a temperature correction formula derived from static loading test conducted at various temperatures in a laboratory maintained with a constant ambient temperature. In this study, the experimental temperature correction procedure is proposed to obtain temperature correction formula by performing the test simulating the field conditions as possible using an apparatus conceived to maintain a constant load level. Figures 6 and 7 illustrates the testing apparatus enabling to maintain a constant load and to apply loading on the bearing with capacity of $1000 \mathrm{kN}$ without particular equipment. This apparatus is identical to the test setting used for the fatigue test in Chapter 2 except the absence of the actuator used to apply the additional dynamic load. Compared to the testing apparatus of Chapter 2, the capacity of the hydraulic nut is increased to enable loading by means of a hydraulic jack with capacity larger than $1000 \mathrm{kN}$. The apparatus was fabricated to prevent as possible the occurrence of eccentricity. This apparatus allows static loading test using a hydraulic jack and also to apply constant loading by fixing the hydraulic nut $[4,5]$.

The specimen adopted for the test is the load-measur- 


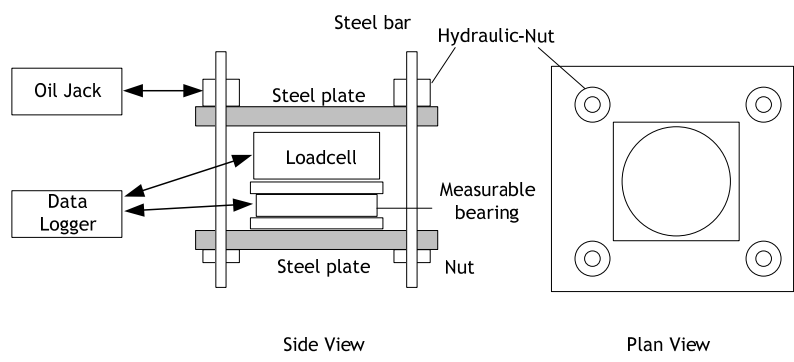

Figure 6. Setting of temperature correction test.

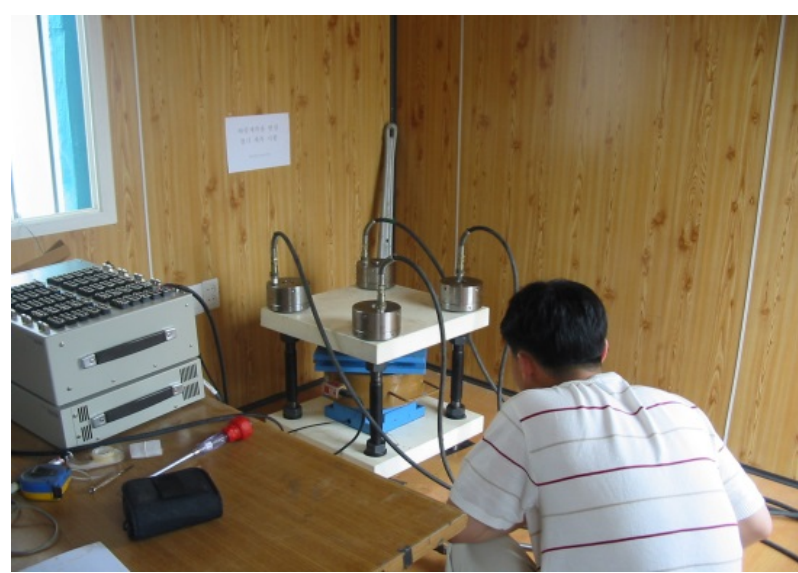

Figure 7. Hydraulic loading during the temperature correction test.

ing pot bearing with capacity of $1000 \mathrm{kN}$ fabricated with identical dimensions and characteristics to $1 \mathrm{MN}-\mathrm{L} 1$ of Part I. For the load-measuring pot bearing proposed in Part I, static loading test shall be performed on all the fabricated bearings to obtain the applied load and the conversion formula of the built-in load cell. To go further, loading test shall be re-executed when the bearings are reassembled since the contact conditions between the button of the built-in load cell and the elastomer disk inside the bearing undergo changes. Accordingly, static loading test was conducted by loading the fabricated bearing by the hydraulic jack using the test apparatus described in Figure 6. Differently to the test using the actuator in Part I, the loading speed is not constant in this test because loading is applied by a hydraulic jack in which the hydraulic pressure is controlled manually. Figure 8 plots the test results and the conversion formula is as follows.

$$
F=456.2208 f-66.4447,
$$

where $f$ is the load $(\mathrm{kN})$ measured by the $5000 \mathrm{kN}$-load cell inserted in the bearing; and, $F$ is the vertical load $(\mathrm{kN})$ applied on the bearing that is the load converted from the measured load. Equation (2) was obtained through linear regression analysis limited to the loads larger than $30 \%$ of the capacity of the bearing as explained in Part I. The maximum error for load level lar-

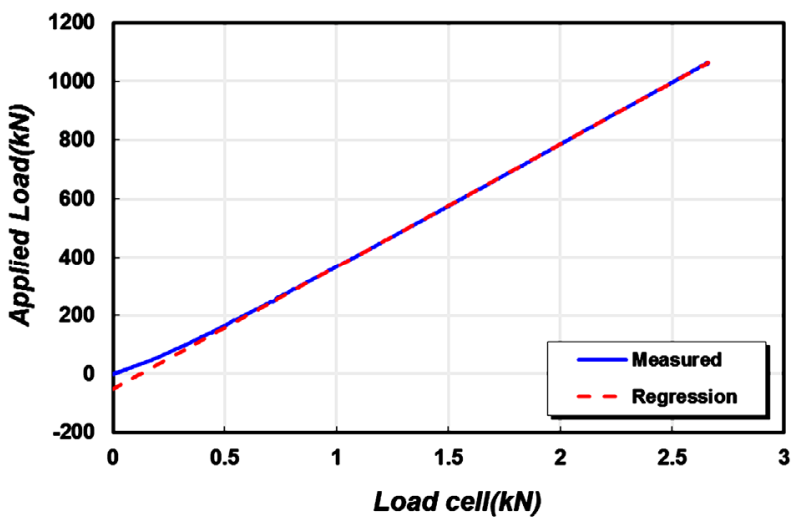

Figure 8. Static loading test and computation of conversion formula.

ger than $30 \%$ is $0.25 \%$. The test was performed at ambient temperature of $31.3^{\circ} \mathrm{C}$.

Temperature correction test was conducted by maintaining the load at $1000 \mathrm{kN}$ using the hydraulic jack and fastening the hydraulic nut. The signals of the load cell measuring the load applied externally, the signals of the built-in load cell and the temperature were measured with respect to time. Slight change of the load from the constant value of $1000 \mathrm{kN}$ occurred due to temperatureinduced changes encountered by the steel bars, bearing and load cells. Figure 9 plots the temperature history, the externally applied load history and the load history obtained by conversion of the measurement of the built-in load cell. It can be observed that all the histories exhibit similar trend. However, the error history between the external load history and measured load history reaches $15 \mathrm{kN}$ and shows regular pattern with respect to the temperature history. Consequently, need is to correct this error history.

In order to correct the error with respect to the temperature history, need is to examine the factors causing such error. To that goal, the temperature history and loading history and, the temperature history and measured load history were superposed and magnified as shown in Figure 10. In Figure 10(a), the external load history and the temperature history exhibit roughly similar trend but a slight time lag can be observed as well as some difference in the shape of the sine wave. Such differences can be attributed to the characteristics of the hydraulic nut fixing the steel bars and the elastomer disk inside the bearing. Besides, in Figure 10(b), the measured load history obtained by conversion of the load history of the small size load cell does not show time lag in comparison to the thermal load history and their shapes are also in good agreement. This good correspondence can be explained by the change of the contact area with the elastomer disk owing to the instantaneous deformation of the built-in load cell itself. To summarize, the error according to the temperature history of the meas- 


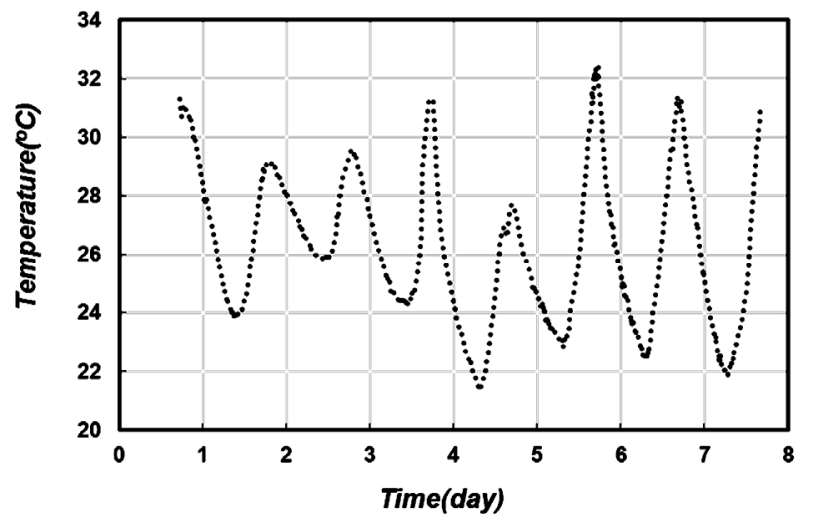

(a)

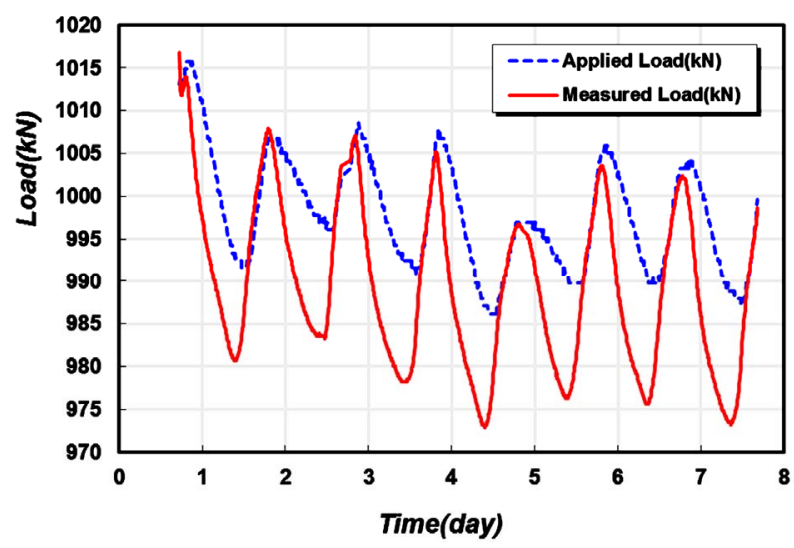

(b)

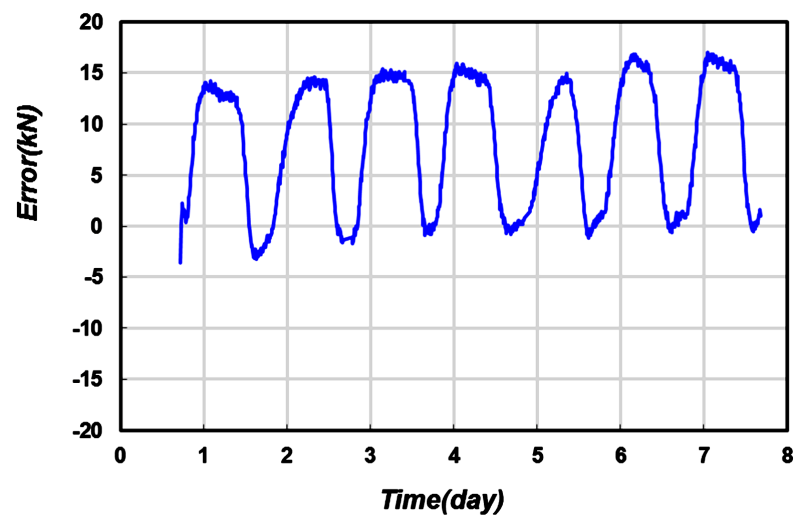

(c)

Figure 9. Temperature correction test results: (a) Temperature history; (b) Load history; (c) Error history.

ured and applied load histories is caused to the instantaneous deformation of the built-in load cell with respect to the temperature, which modifies the degree by which the load cell is inserted in the flexible elastomer disk and, in turn, changes the contact area between the load cell and the elastomer. Based upon this discussion, the conversion formula enabling to correct the temperature history is modified and is proposed as follows.

$$
F(t)=[A+a \Delta T] f\left(t+t_{0}\right)+C
$$

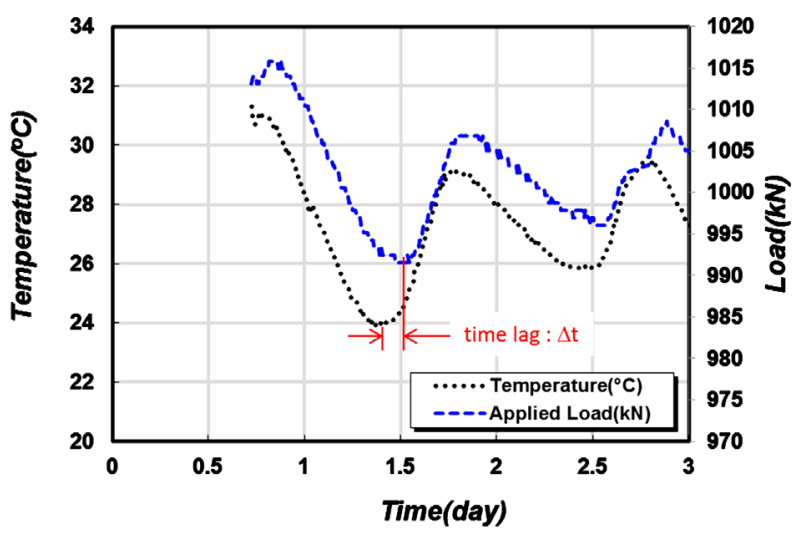

(a)

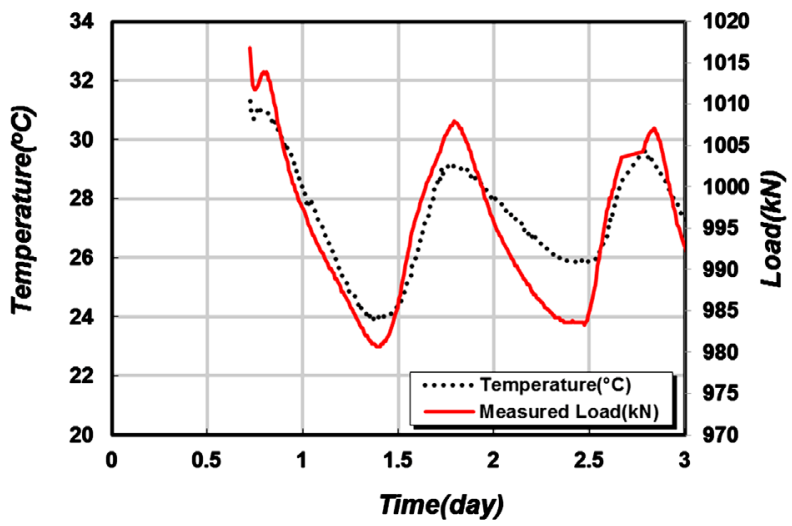

(b)

Figure 10. Comparison of temperature and loading histories: (a) Temperature history and loading history; (b) Temperature history and measured load history.

where $A$ and $C$ are constants that can be obtained from the static loading test, here $A=418.93$ and $C=-51.79$. These values are calculating assuming that the constants are maintained at definite temperature and during a period longer than a definite time. In Equation (3), $t_{0}$ is the time lag constant caused by the characteristics of the elastomer disk determined in the long-term measurement test. In the figure, $t_{0}=0.11$ day. The constant $a$ has value representing the change in the contact area according to the characteristics of the elastomer disk and temperature change in the long-term measurement test and can be obtained by linear regression analysis. Here, $a=-0.573$. Finally, the conversion formula can thus be rewritten as

$$
F(t)=[418.93-0.573 \Delta T] f(t+0.11)-51.79
$$

Figure 11 plots the history of the measured load calculated using the conversion formula corrected with respect to the temperature history, the history of the applied load and the corresponding error history. It can be seen that the error experiences significant reduction from $15 \mathrm{kN}$ before correction to $10 \mathrm{kN}$ after correction. 


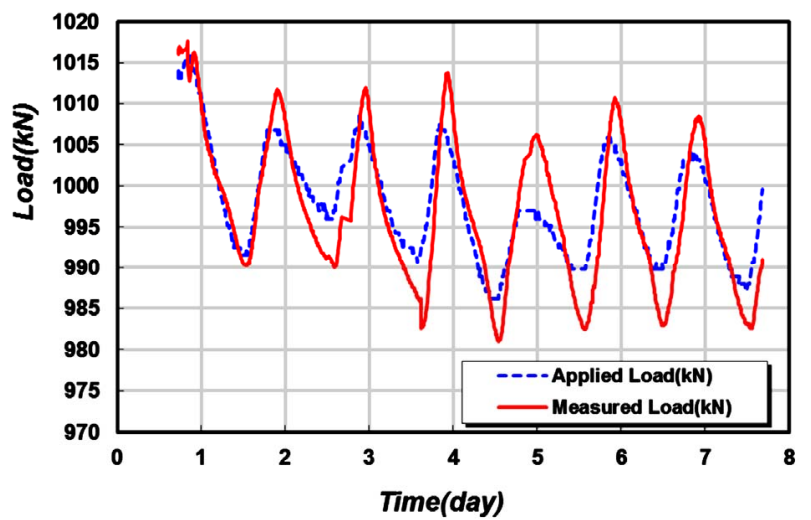

(a)

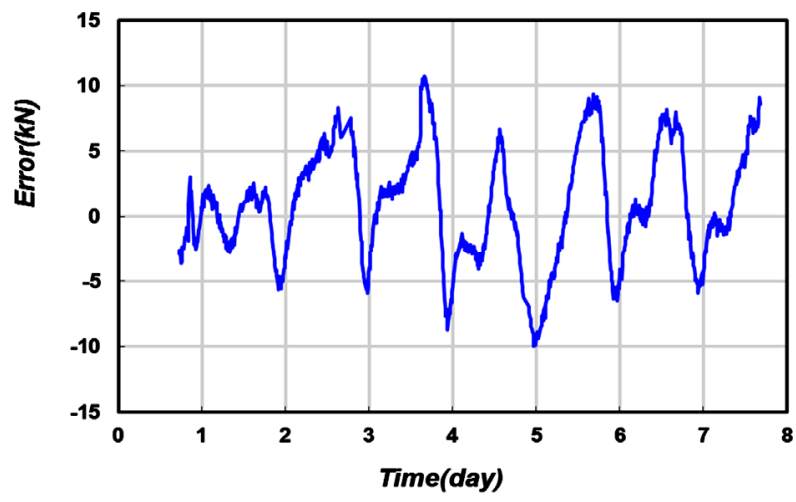

(b)

Figure 11. Test results when applying the corrected conversion formula: (a) Applied load history and corrected measured load history; (b) Error history when applying the corrected conversion formula.

\section{Conclusion}

Even if the measurement accuracy tended to degrade gradually with the accumulation of fatigue, the degree of accuracy was verified to be sufficient for the intended application. The error history of the developed bearing exhibited regular variation according to the temperature history. The application of the conversion formula obtained by linear regression analysis of the external load and the load measured by the built-in load cell in the static loading test resulted in an error reaching $1.5 \%$ to $2.0 \%$ of the total capacity of the bearing. Moreover, the cause of such error could be attributed to the instantane- ous deformation of the built-in load cell with respect to the temperature, which modified the extent by which the load cell was inserted in the flexible elastomer disk and, in turn, changed the contact area between the load cell and the elastomer. Accordingly, the experimental temperature correction procedure is proposed to reduce the temperature induced error. The modified conversion formula from the procedure enabled to reduce the error to approximately $1 \%$. Outstanding sensitivity and accuracy of measurement could be verified through the fatigue tests. The load-measuring pot bearing with built-in load cell was seen to exhibit sufficient field applicability under application of the proposed temperature correction formula.

\section{Acknowledgements}

This research was supported by a grant from a Strategic Research Project (Development of smart prestressing system for prestressed concrete bridges) funded by the Korea Institute of Construction Technology.

\section{REFERENCES}

[1] J.-R. Cho, Y. J. Kim, J.-W. Kwark, S.Y. Park, W. J. Chin and B.-S. Kim, "Load-Measuring Pot Bearing with Built-in Load Cell-Part I: Design and Performance," Engineering, Scientific Research Publishing, 2013 (under Review).

[2] Ministry of Land, Transport and Maritime Affairs, Highway Bridge Design Code, 2010, in Korean.

[3] Korea Institute of Construction Technology, "R\&D on the Safety of High-Speed Railway Structures,” R\&D Report, 2002, in Korean.

[4] Korea Institute of Construction Technology, "Development of Stabilization Techniques for Track Construction System of the High-Speed Railway,” R\&D Report, 2003, in Korean.

[5] Korea Institute of Construction Technology, "Development of Stabilization Techniques for Track Construction System of the High-speed Railway,” R\&D Report, 2004, in Korean. 